\title{
EVALUATION DES TAUX DE RECAPTURE ET DE RETOUR DE LA TRUITE DE MER SUR LE BASSIN DE LA BRESLE (HAUTE-NORMANDIE / PICARDIE)
}

\author{
Françoise FOURNEL, G. EUZENAT, J.L. FAGARD \\ Conseil Supérieur de la Pêche \\ Délégation Régionale $n^{\circ} 1$ - 3, rue Ste Marie - 60200 COMPIËGNE \\ Collaboration technique
}

- Brigade Mobile d'Intervention (C.S.P. / DR 1)

- Brigades de garderie des Fédérations des A.A.P.P. de la Somme et de la Seine-Maritime.

\section{RÉSUME}

Plusieurs campagnes de marquage ont été réalisées de 1982 à 1988 sur le bassin de la BRESLE visant à définir, en conditions naturelles, les taux de recapture et de retour de la truite de mer, dans le cadre de l'étude fonctionnelle des populations en cours sur ce bassin.

Les poissons sont marqués

- soit au stade smolt : cinq campagnes de marquage réalisées de 1982 à 1986, 17.410 poissons marqués au total

- soit au stade adulte bécard : trois campagnes réalisées, 481 truites de mer marquées.

Deux types de marquage sont utilisés :

- marquage de groupe par tatouage à l'azote liquide, accompagné d'une ablation de la nageoire adipeuse, pour la plus grande partie des smolts

- marquage individuel par ètiquette Carlin avec attache par fil polyéthylène. pour les smolts restants et pour tous les bécards

Une attention particulière est portée à la récupération des poissons marqués (piègeage et captures par pêche, en milieu fluvial et maritime côtier)

Les retours de marques sont issus d'une vaste zone géographique mais proviennent pour l'essentiel de la Bresle et de la zone maritime environnante (dispositif de piègeage et filets fixes côtiers).

Pour les smolts, les taux de recapture (chiffres bruts) sont compris entre 4,4 et $12,3 \%$, les taux de retour entre 5,5 et $20 \%$

Les valeurs observées sont toujours plus élevées pour les poissons cryomarqués que pour ceux marqués Carlin (taux de retour à la zone de départ de $15 \%$ en moyenne pour les premiers, de $9,2 \%$ pour les seconds).

Les taux de recapture et de retour "truite de mer" sont toujours supérieurs, d'un facteur 1,7 à 2,2 selon le taux considéré, aux taux "saumon" obtenus dans les mêmes conditions.

Les taux observés chez les bécards (taux de retour à la zone de départ compris entre 31 et $50 \%$ ) témoignent de l'excellente survie post-frai des géniteurs de truite de mer.

Les travaux réalisés ont permis d'obtenir les premiers chiffres français sur les taux de recapture et de retour de Salmonidés migrateurs sauvages; ils constituent à ce titre une étape importante dans la connaissance du fonctionnement dynamique de leurs populations.

\section{ESTIMATION OF RECAPTURE AND RETURN RATES OF SEA-TROUT ON BRESLE RIVER, UPPER NORMANDY / PICARDY}

\section{SUMMARY}

From 1982 to 1988 , several marking operations have been set on Bresle river, in order to estimate the recapture and return rates of wild sea-trout; these investigations were integral part of the study on salmonid population dynamics conducted on this river

Fish have been marked after downstream trapping as smolts or as just-spawned, mended or not. As a whole, 15.100 smolts were cold-branded and adipose-fin clipped; 2.310 smolts and 481 kelts were Carlin tagged with double polyethylen thread. 
Considerable attention was paid to recapture, operated by upstream trapping and control of marked returning fish in coast nets and rod-and-line fisheries.

Marks returns came from a large area as far as Danemark coast, but mainly from Bresle river trapping facilities and fixed nets on next coast.

The gross recapture rate of wild sea trout smolts is between 4,4 and $12,3 \%$; their return rate range from 5.5 to $20 \%$.

Branded fish always give higher return rates than Carlin tagged ones : $15 \%$ average to $9,2 \%$; sea trout survive 1,7 to 2,2 better than salmon.

Kelt sea survival is good as indicated by their high return rate : 31 to $50 \%$ according to years.

These leading french results about wild sea-trout survival contribute greatly to the knowledge of salmonid population dynamics.

\section{INTRODUCTION}

Depuis 1982, la dynamique de population de la truite de mer est étudiée sur le bassin de la Bresle, petit fleuve côtier de $66 \mathrm{~km}$ de longueur formant la frontière entre les départements de la Somme et de la Seine-Maritime. Des structures de contrôle des migrants y ont été mises en place, permettant d'aboutir à une connaissance quantitative et qualitative des entrées (adultes reproducteurs) et des sorties (smolts et bécards) sur plusieurs années consécutives.

Les expériences de marquage / recapture faisant l'objet de la présente publication ont été réalisées dans le cadre de cette étude fonctionnelle; elles visent à définir deux des paramètres qui régissent l'importance des populations : taux de recapture et taux de retour à la rivière d'origine des migrateurs à l'issue de leur séjour en mer. Elles concernent donc excluvivement des poissons sauvages.

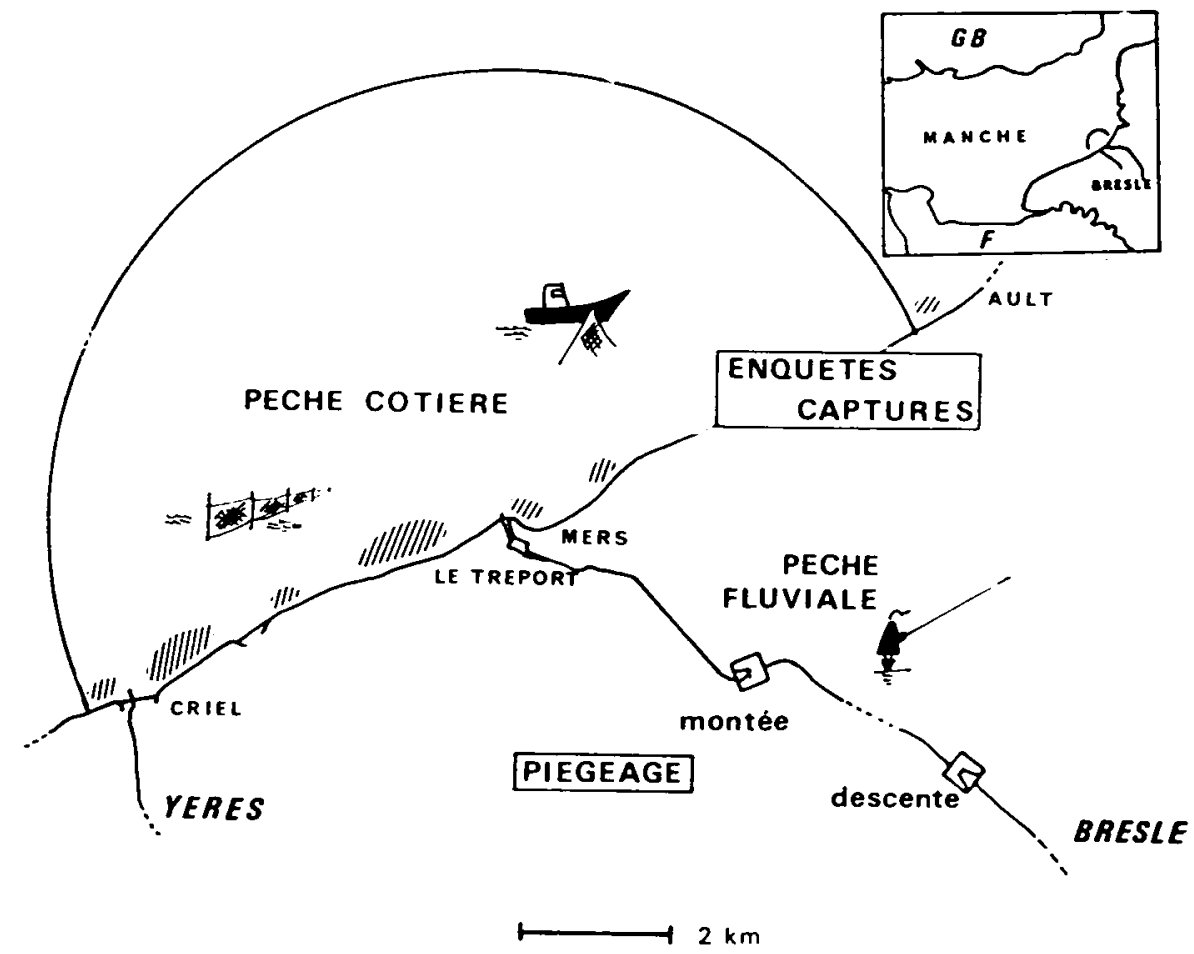

figure 1 : Infrastructure d'étude et de récupération des marques mise en place sur le bassin de la BRESLE (Haute-Normandie/Picardie). Localisation des dispositifs de piègeage et des principaux sites d'enquête dans le périmètre rapproché (rayon de $6 \mathrm{~km}$ autour de l'embouchure). En hachuré, les pêcheries cótières (filets fixes sur plage).

figure 1 : Diagram of the study area on Bresle river and on the coast near the river mouth (6 $\mathrm{km}$ radius). Location of smolt and adult traps and catch survey sites. Slanted : coastal fisheries (stake nets). 


\section{LIEUX ET MÉTHODES D'ÉTUDE}

\subsection{Marquage}

Il est pratiqué uniquement sur des poissons de descente, capturés par piègeage. Le dispositif de capture est implanté sur la rivière Bresle, à $15 \mathrm{~km}$ de la mer (dispositif du Lieu-Dieu, commune de Beauchamps, dans la Somme - figure 1).

Les poissons sont marqués:

- soit au stade smolt ou présmolt : cinq campagnes de marquage ont été réalisées, de 1982 à 1986. Les smolts sont majoritairement âgés de 1 an; ils mesurent en moyenne $195 \mathrm{~mm}$ pour un poids de 80 g. (Figure 2)

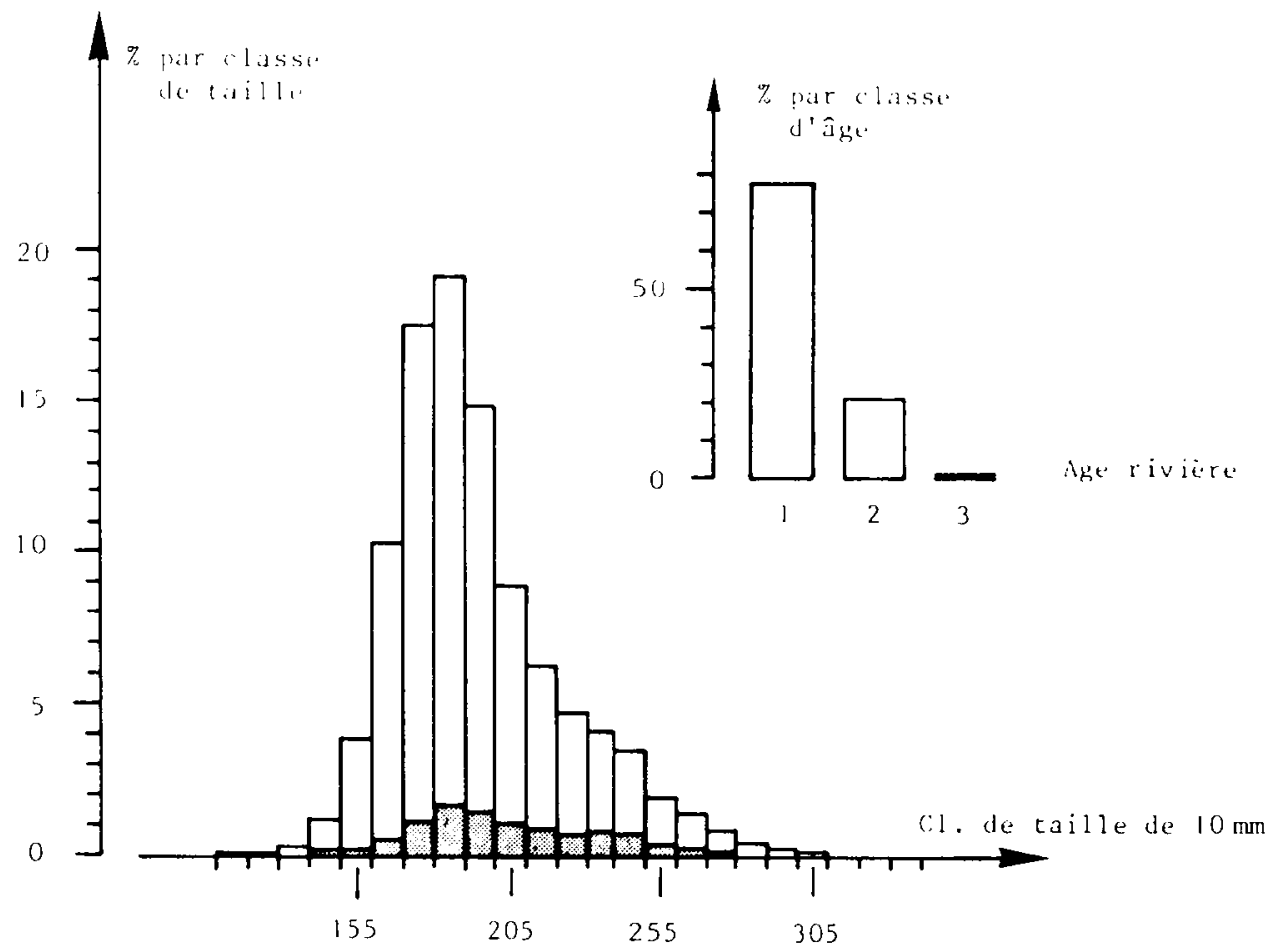

figure 2 : Structures de taille et d'âge des smolts de truite de mer marqués sur la Bresle lors de leur dévalaison. Dispositif de contrôle du Lieu-Dieu — Printemps 1982 à 1986 (en grisé, smolts marqués par étiquette Carlin).

figure 2 : Size and age trequency distribution of sea-trout smolts caught in Lieu-Dieu downstream trap on Bresle river, 1982-1986.

Carlin-tagged fish : darked area

Branded fish : clear area.

- soit au stade bécard : trois campagnes réalisées, de 1985 à 1988. Le terme bécard désigne ici les adultes d'avalaison, ayant frayé, quel que soit leur état de récupération physiologique.

Deux types de marquage sont utilisés :

- pour la majorité des smolts, un marquage de groupe par tatouage à l'azote liquide

- pour une fraction des smolts et tous les bécards, un marquage individuel par étiquette Carlin.

Au total, le marquage a porté sur 17.410 smolts et 480 bécards. Le détail, par année et par type de marque, est donné dans le tableau 1.

Pour le tatouage à l'azote liquide, le fer employé est en maillechort et présente les caractéristiques suivantes : hauteur et largeur : $10 \mathrm{~mm}$, empatement : $16 / 10 \mathrm{~mm}$; il est appliqué pendant deux secondes (temps rythmé au métronome) sur l'épaule du poisson préalablement anesthésié, mesuré et éventuellement pesé. 
Tableau I : Taux de recapture et de retour des différentes cohortes de truites de mer marquées de 1982 à 1986 - Rivière BRESLE (76 - 80)

A - Smolts sauvages cryomarqués

B - Smolts sauvages - étiquettes Carlin

(1) tous moyens de capture

(2) périmètre rapproché

(3) résultats partiels : retours 88 non pris en compte.

Table I : Recapture and return rates of five annual sea-trout smolt runs in Bresle river.

A - cold-branded fish

B - Carlin-tagged fish

(1) all catching methods

(2) in the near area

(3) part data : 1988 returns are not included.

A

\begin{tabular}{|c|c|c|c|c|c|}
\hline ANNEES MARQUAGE & 1982 & 1983 & 1984 & 1985 & $1986^{(3)}$ \\
\hline NOMBRE SMOLTS MARQUES & 2700 & 3910 & 1030 & 3805 & 3653 \\
\hline TOTAL RECAPTURE (1) & 181 & 354 & 104 & 468 & $(313)$ \\
\hline $\begin{array}{l}\text { PIEGE MONTEE EU } \\
\text {. Eff. capturés } \\
\text {. Eff. estimés }\end{array}$ & $\begin{array}{l}118 \\
195 \\
\end{array}$ & $\begin{array}{l}246 \\
398 \\
\end{array}$ & $\begin{array}{r}79 \\
112 \\
\end{array}$ & $\begin{array}{l}262 \\
411 \\
\end{array}$ & $\begin{array}{l}(153) \\
(256)\end{array}$ \\
\hline $\begin{array}{l}\text { CAPTURES PAR PECHE (2) } \\
\text {. Eff. déclarés } \\
\text {. Eff. estimés }\end{array}$ & $\begin{array}{r}51 \\
112 \\
\end{array}$ & $\begin{array}{r}74 \\
163 \\
\end{array}$ & $\begin{array}{l}19 \\
42 \\
\end{array}$ & $\begin{array}{l}158 \\
348 \\
\end{array}$ & $\begin{array}{r}(88) \\
(\underline{194}) \\
\end{array}$ \\
\hline TAUX DE RECAPTURE BRUT & $6,7 \because$ & $9,05 \because$ & $10,1:$ & 12,3 & $(8,6:)$ \\
\hline $\begin{array}{l}\text { TAUX DE RETOUR } \\
\text { A LA ZONE DE DEPART }\end{array}$ & 11,4 & 14,3 & 14,9 & 19,9 & $(12,3 ;)$ \\
\hline $\begin{array}{l}\text { TAUX DE RETOUR } \\
\text { AU PIEGE }\end{array}$ & 7,2 & 10,2 & 10,9 & 10,8 & $(7)$ \\
\hline
\end{tabular}

B

\begin{tabular}{|l|c|c|c|c|c|}
\hline ANNEES MARQUAGE & 1982 & 1983 & 1984 & 1985 & $1986(3)$ \\
\hline NOMBRE SMOLTS MARQUES & 493 & 498 & 258 & 510 & 553 \\
\hline TOTAL RECAPTURE (1) & 1 & 22 & 19 & 42 & $(47)$ \\
\hline $\begin{array}{l}\text { PIEGE MONTEE EU } \\
\text { Eff. capturés } \\
\text { Eff. estimés }\end{array}$ & 1 & 10 & 10 & 19 & $(25)$ \\
\hline $\begin{array}{l}\text { CAPTURES PAR PECHE (2) } \\
\text { Eff. déclarés } \\
\text { Eff. estimés }\end{array}$ & - & 15 & 13 & 29 & $(42)$ \\
\hline $\begin{array}{l}\text { TAUX DE RECAPTURE BRUT } \\
\text { TAUX DE RETOUR } \\
\text { A LA ZONE DE DEPART }\end{array}$ & - & 7,4 & 7,4 & 8,2 & $(15)$ \\
\hline $\begin{array}{l}\text { TAUX DE RETOUR } \\
\text { AU PIEGE }\end{array}$ & - & 5,4 & 8,1 & 17 & $(25)$ \\
\hline
\end{tabular}


Les trois lettres $X, O$ et $H$ sont utilisées en rotation sur trois ans.

Le tatouage est toujours accompagné d'une ablation de la nageoire adipeuse

Marquage par étiquette Carlin : le poisson, préalablement anesthésiè, mesuré et pesé, est immobilisé dans un conteneur à revêtement intérieur souple. La marque, montèe sur fil polyéthylène, est mise en place par deux opérateurs, à l'aide d'un porte-aiguilles. Le serrage du nœud (double nœud croisé) est assuré à la pince. A signaler toutefois que deux éléments singularisent la campagne 82 : la nature du fil, moins souple et plus fragile que celui utilisé par la suite et le serrage du nœud, réalisé sans pince la première année.

\subsection{Récupération des poissons marqués (Figure 1)}

\section{Elle s'effectue}

- dans le dispositif de contrôle des poissons d'amontaison installé sur la Bresle à Eu, à $3 \mathrm{~km}$ de la mer

- auprès des pêcheurs, à la ligne en rivière et au filet en mer (pêche à pied, au filet fixe sur plage et pêche en bateau, au filet maillant)

Un effort important d'information/sensibilisation a été déployé dans les quatre départements côtiers concernés (Eure, Seine-Maritime, Somme et Pas-de-Calais) mais plus particulièrement sur la "zone BRESLE", englobant le cours inférieur de la rivière et la zone côtière dans un rayon de $6 \mathrm{~km}$ autour de son embouchure (sites de Criel, Mers et Le Tréport).

Cet effort d'information s'est assorti d'une forte présence sur le terrain : passage régulier d'enquêteurs sur les principaux sites de pêche (une fois par semaine au minimum pendant toute la saison de pêche), suivi des captures, grande disponibilité pour les constats de marques,... et d'une incitation financière: prime de $25 \mathrm{~F}$ pour toute déclaration de marque exploitable, remise de prix par tirage au sort en fin de saison (1.000 francs au premier). Démarrées depuis 1978 sur le site de Criel, les enquêtes n'ont débuté qu'en 1984 sur le site de Mers/Le Tréport pour s'intensifier en 1986 et 1987.

Des contacts ont en outre été établis avec les Services des Pêches des pays côtiers voisins : Belgique, Pays-Bas, Angleterre et Danemark.

- et enfin lors d'opérations techniques ponctuelles : capture des bécards dans le dispositif de contrôle du Lieu-Dieu; pêches électriques de récupération des géniteurs au pied des obstacles difficilement franchissables sur les bassins de la Canche, de l'Authie et de l'Arques (Figure 3).

\subsection{Evaluation des facteurs correctifs}

Pour dépasser les chiffres bruts de recapture et tenter d'approcher au mieux les taux de retour réels des poissons marqués, une évaluation a été faite

- de l'efficacité du dispositif de piègeage des adultes implanté à EU. Pour cela, tous les poissons capturés à la montée sont systématiquement marqués par section du tiers d'une nageoire pelvienne, droite ou gauche une année sur deux. Le contrôle des sections se fait sur les géniteurs qui redescendent vers la mer après le frai, ceux-ci étant capturés de Décembre à Avril dans le dispositif de contrôle du Lieu-Dieu.

Quatre campagnes de contrôle ont été réalisées portant sur les remontées 84 à 87 ; l'efficacité du dispositif varie selon les années de 59 à $70 \%$, soit un facteur correctif compris entre 1,4 et 1,7. A signaler que pour l'année 83, la correction a été faite sur cette même base alors que la perméabilité du dispositif était plus importante (les grilles de guidage du poisson n'ayant été complètement installées qu'au début de 1984).

- du taux de déclaration, par les pêcheurs, des captures de poissons marqués.

Le calcul d'un facteur correctif n'a été réalisé que pour la zone rapprochèe, là où l'exploitation par pêche fait l'objet, depuis 1986, d'un contrôle soutenu. Il s'appuie sur:

- l'évaluation du volume annuel des captures sur les différents sites de pêche, faite à partir des enquêtes sur le terrain et des déclarations des pêcheurs (carnets de pêche).

- la proportion de poissons marqués observée dans les captures au piège ( $32 \%$ en moyenne) et dans les captures des pêcheurs contrôlées par les enquêteurs ( $30 \%$ en moyenne)

- les proportions, dans les recaptures issues des pêcheurs, de celles ayant fait l'objet d'une déclaration volontaire d'une part, de celles constatées lors des enquêtes d'autre part; ainsi, sur la pêcherie la plus proche du débouché de la Bresle, ces proportions sont-elles de 35 et $65 \%$ respectivement alors que les enquêtes ne couvrent que $15 \%$ des marées en moyenne! 
Les différents recoupements réalisés permettent de penser que les taux de déclaration n'excèdent pas:

- $45 \%$ pour les cryomarques (moyenne calculée sur les années 86 et 87 ), soit un facteur correctif minimal de 2,2

- $58 \%$ pour les étiquettes Carlin, soit un facteur correctif minimal de 1,7 .

Le meilleur taux de déclaration des étiquettes Carlin est attesté par la proportion, dans les retours totaux, des retours issus des pêcheurs : si les pêcheurs fournissent, en moyenne, $34 \%$ des retours de cryomarque, cette proportion passe à $45 \%$ pour les étiquettes.

En l'absence de moyens d'évaluation, le mème facteur de correction a été appliqué aux chiffres des années 83 à 85 , moins bien suivies pourtant sur le plan de l'exploitation ; il y a donc, pour ces trois années, sous-évaluation probable des retours.

Aucun facteur correctif n'a étè appliqué aux captures éloignées (sortant de ce périmètre de 6 $\mathrm{km})$ qui, de toute manière, ne sont pas prises en compte pour l'évaluation des taux de retour.

\subsection{Définition des termes employés}

- Taux de recapture brut : effectifs des poissons marqués repris au cours des années $n, n+1$, $n+2, \ldots$ par tous moyens de capture sur l'ensemble de la zone de recapture, rapportés aux effectifs initiaux. Ce taux ne subit aucune correction.

- Taux de retour à la zone de départ : effectifs estimés des poissons marqués atteignant la BRESLE ou ses environs immédiats (rayon de $6 \mathrm{~km}$ autour de l'embouchure), rapportés aux effectifs initiaux.

- Taux de retour au piège : effectifs estimés de poissons marqués qui reviennent jusqu'au dispositif de piègeage, rapportés aux effectifs initiaux. II correspond donc au retour à la zone de départ moins les prélèvements par pêche, en mer et en rivière, à l'aval du dispositif (Figure 1).

\section{RÉSULTATS}

\subsection{Localisation des recaptures}

Les retours de marque sont issus d'une vaste zone géographique qui s'étend du Havre au nord du Danemark, sur un front de l'ordre de $1000 \mathrm{~km}$, mais ils proviennent à $96 \%$ de la BRESLE et de la zone maritime proche (rayon de $6 \mathrm{~km}$ autour de son embouchure) (Figure 3).

II est à signaler que les retours lointains, $100 \mathrm{~km}$ et plus, concernent exclusivement des étiquettes Carlin, en dépit de l'information réalisée et de la présence, dans tous les départements, de relais bien connus des pêcheurs pour la récupération de l'information (gardes-pêche et gendarmes maritimes).

\subsection{Répartition des recaptures en fonction des modes de capture}

Les retours de marques proviennent pour l'essentiel :

- du dispositif de contrôle des poissons d'amontaison : $60.3 \%$ des recaptures

- des déclarations des pêcheurs en mer : $29,3 \%$, principalement des amateurs aux filets fixes côtiers opérant sur les sites voisins du débouché de la Bresle (Mers / Le Tréport et Criel-sur-Mer).

II est d'ailleurs intéressant de noter à ce propos :

- que la proportion des retours de marques issus de cette catégorie augmente sensiblement au cours de la période d'étude, passant de $20 \%$ en moyenne en $1984 / 85$ à $34 \%$ en moyenne à partir de 1986, en relation avec un effort plus important d'enquête et d'information

- que cet accroissement concerne surtout les retours de cryomarques (19 à 33,5\%), les retours d'étiquettes Carlin étant moins influencés par l'intensification des enquêtes ( 33 à $37 \%$ ).

Les retours de marques issus des pêcheurs à la ligne restent faibles : $3.1 \%$ en moyenne, cette faiblesse s'expliquant par un effort de pêche et une efficacité de capture nettement inférieurs à ceux des pêcheurs côtiers.

Quant aux $7,3 \%$ imputables aux interventions techniques diverses, ils proviennent en presque totalité du dispositif de contrôle des poissons d'avalaison du Lieu-Dieu implanté sur la Bresle, les captures de poissons marqués étant tout à fait fortuites lors des opérations ponctuelles réalisées sur d'autres cours d'eau.

\subsection{Taux de recapture et de retour à partir des smolts}

Les principaux résultats des cinq campagnes de marquage effectuées sur les smolts de 1982 à 1986 sont présentés dans le tableau 1. 


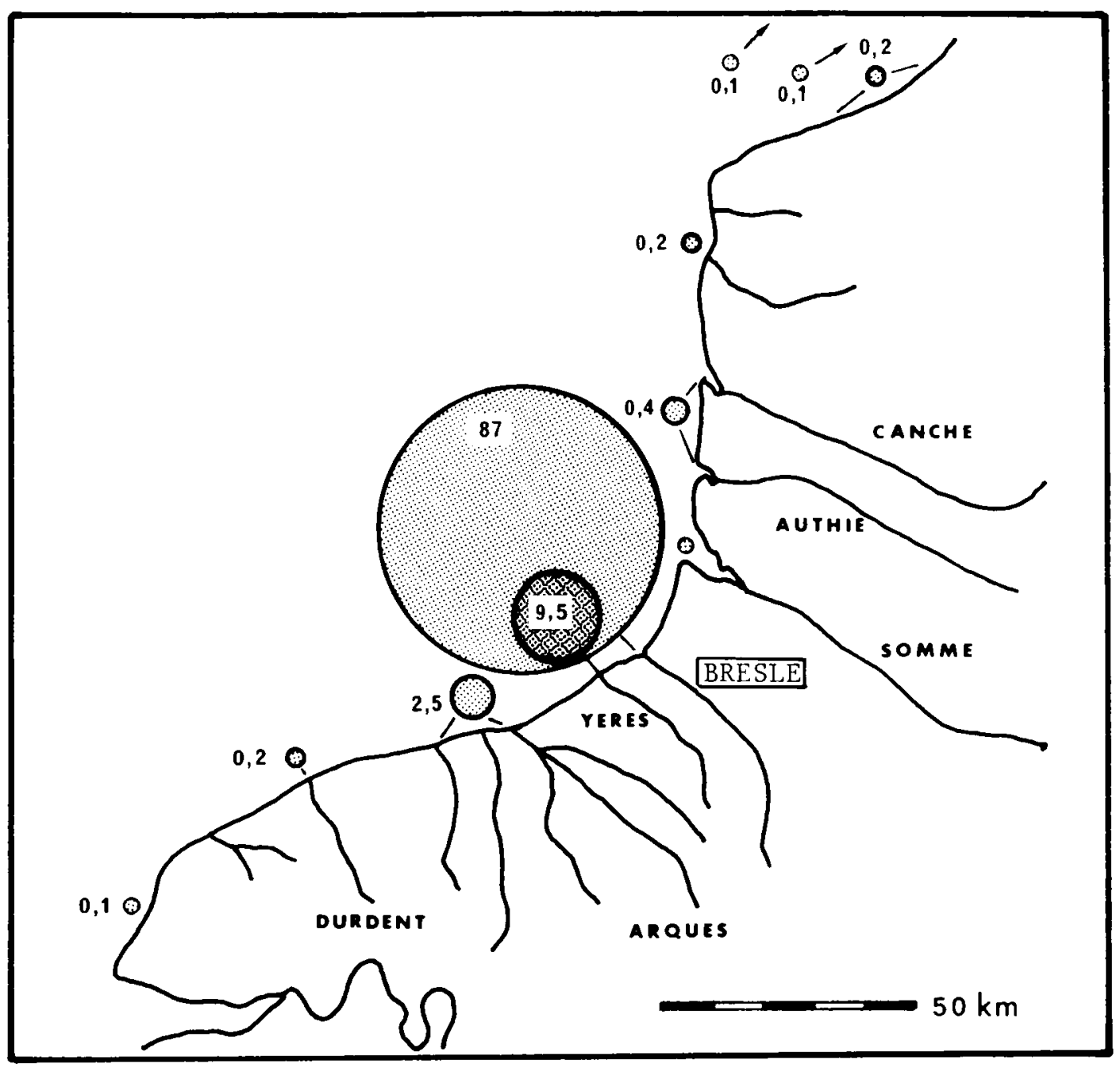

figure 3 : Eclatement géographique des recaptures de truites de mer marquées sur la Bresle au stade smolt ou adulte bécard. Les chiffres accompagnant les cercles représentent les pourcentages calculés sur les années 83 à 87 (chiffres bruts).

figure 3 : Spread recapture sites of sea-trout smolts and kelts marked on Bresle river. Numbers are gross percentages during the 1983-87 period.

Compte tenu des imperfections, apparemment rédhibitoires du marquage par étiquette Carlin en 1982 (cf. paragraphe 2.1), les résultats quasi-nuls de cette première campagne n'ont pas été intégrés dans le calcul des moyennes.

- Les taux de recapture bruts s'établissent à $9,5 \%$ en moyenne pour les smolts cryomarqués $(6,7$ à $12,3 \%)$ à $7,1 \%$ pour les smolts marqués par étiquette Carlin (4,4 à $8,5 \%)$; ils sont en constante progression sur la période $82 / 86$. retour.

La perte de marque intervient évidemment pour faire baisser les taux de recapture et de

Grâce au double marquage pratiqué, le taux d'effacement des cryomarques a pu être évalué: il s'élève à $5,7 \%$ sur les poissons contrôlés au piège, à $16,9 \%$ sur les poissons pris au filet. L'effacement du tatouage est la plupart du temps lié, y compris pour les poissons piègés, à l'écaillage par les filets de la partie antérieure du corps.

Aucune évaluation de la perte de marques n'a été faite pour le marquage Carlin, pratiqué seul.

Pour ce mode de marquage, on observe des différences importantes de taux de recapture selon la taille au marquage, celui-ci passant de 3,6 \% en moyenne pour les smolts de taille inférieure à $22 \mathrm{~cm}$, à 13,2\% pour ceux de taille supérieure à $22 \mathrm{~cm}$; soit un facteur de 3,7 entre ces deux groupes de taille, qui correspondent sensiblement aux classes d'âge 1 an et 2 ans. 
- Les taux de retour moyens calculés sur les deux groupes, cryomarqués et porteurs d'étiquette Carlin, sont respectivement de:

Tx de retour à la zone de départ

Cryomarquage
$15 \%$
$9,8 \%$

Etiquette Carlin $9,2 \%$

$5,3 \%$

Les résultats détaillés, par année et par mode de marquage, sont présentés Figure 4.

On observe, selon le mode de marquage utilisé, une évolution sensiblement différente des taux de retour sur la période d'étude:

- les valeurs obtenues à partir des smolts cryomarqués restent assez stables pour les cinq cohortes, plus encore en ce qui concerne les retours au piège (les chiffres plus faibles observés pour la cohorte 82 pouvant s'expliquer en grande partie par la moindre efficacité du dispositif de piègeage avant 1984) ; l'augmentation, à partir de 1986, du volume des retours issus des pêcheurs est bien perceptible sur la cohorte 85 , faisant passer le taux de retour à la zone de départ de 14,9 à $19,9 \%$ pour des taux de retour au piège pratiquement similaires.

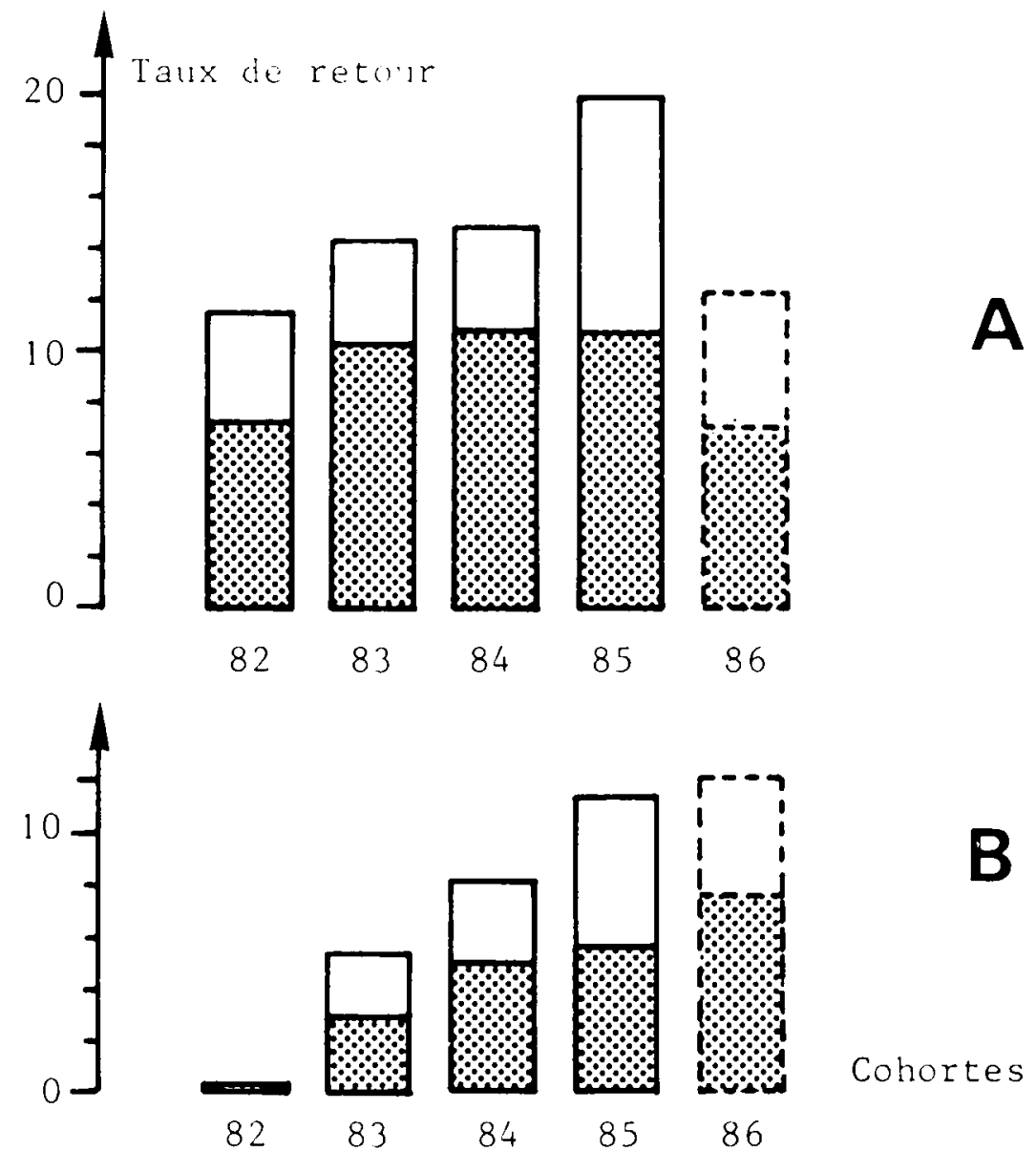

figure 4 : Taux de retour, à la zone de départ et au piège (en grisé), des truites de mer marquées sur la Bresle au stade smolt, de 1982 à 1986.
A - Cryomarquage
B - Etiquettes Carlin.

figure 4 : Return rate of sea-trout smolts marked on Bresle river to the mouth of the river (clear + spotted) to the upstream trap (spotted)

A - Branded fish

B - Carlin-tagged fish. 
- les taux de retour observés à partir des smolts marqués par étiquette Carlin sont en revanche en progression constante : 3 à $7,6 \%$ pour le retour au piège, 5,4 à $12 \%$ pour le retour à la zone de départ, rattrapant même en 1986 les résultats du cryomarquage. Contrairement à ce qui s'observe pour le cryomarquage, la part des retours en provenance des pêcheurs reste pratiquement stationnaire sur toute la période concernée, représentant en moyenne $41 \%$ du total des étiquettes récupérées dans le périmètre "Bresle".

- Taux de premier retour: les taux de retour donnés ici sont des taux globaux, c'est-à-dire intégrant tous les poissons d'une même cohorte revenus aux années $n, n+1, n+2$, etc... après marquage (Figure 5); pour l'essentiel, les recaptures portent sur des individus d'âge $\mathrm{I}^{+}$de mer mais les poissons plus âgés, 11 + et supérieurs, contribuent tout de même pour $24 \%$ aux retours. Certains de ces poissons s'étant déjà reproduits une (ou plusieurs) fois les années précédentes, un même poisson peut être comptabilisé deux fois ou plus dans les retours.

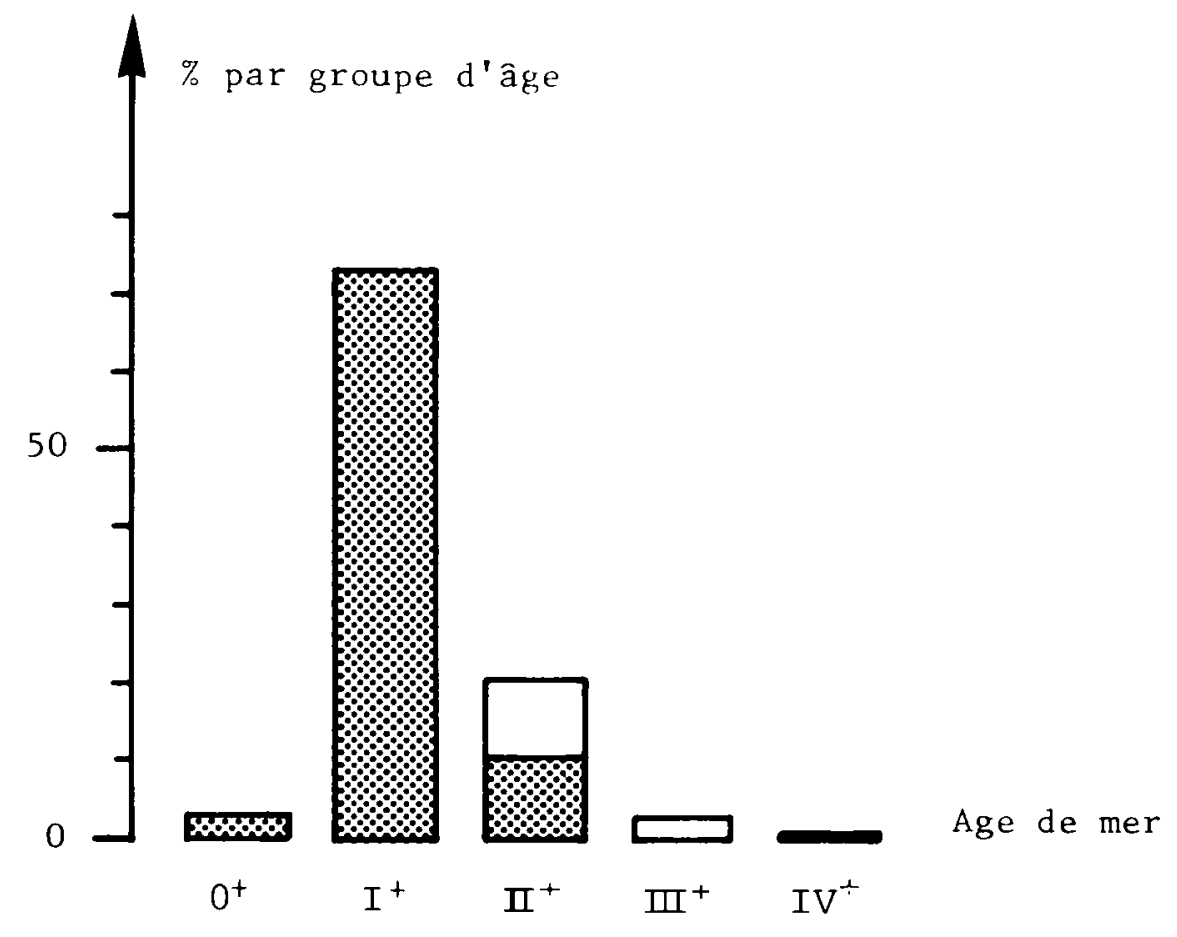

figure 5 : Répartition par groupe d'âge de mer des retours de trultes de mer marquées sur la Bresle au stade smolt (cryomarquage), de 1982 a 1986.

- en grisé : premier retour

- en blanc : retours multiples.

figure 5 : Recapture distribution by sea-age group of sea-trout smolts cold-branded on Bresle river.

- spotted area : mainden fish

- clear area : previous spawners.

Sur le plan dynamique de population, cela n'est pas faux, un poisson qui se reproduit $x$ fois équivalant, pour la rivière, à $x$ poissons. En revanche, pour certaines applications (évaluation de la rentabilité d'une opération de pacage marin par exemple), seul sera à prendre en considération le taux de premier retour.

Au total, compte tenu des âges à la première reproduction qui, sur la Bresle, peut avoir lieu à l'issue de la première, deuxième ou troisième saison de croissance marine (pour $7 \%, 84 \%$ et $9 \%$ de la population respectivement), $15 \%$ seulement des poissons marqués recapturés se sont déjà reproduits au cours du, ou des, précédent(s) hiver(s). Les taux de premier retour, calculés uniquement sur les smolts cryomarqués, s'établissent en conséquence à :

- $13 \%$ en moyenne pour le retour à la zone de départ

- $8,3 \%$ en moyenne pour le retour au piège. 


\subsection{Taux de recapture et de retour à partir des bécards}

Les résultats des expériences de marquage des bécards sont encore incomplets puisque celles-ci n'ont commencé que durant l'hiver 85/86 et que les retours correspondants ne sont pas terminés. Les résultats présentés ici ne concerneront donc que les deux campagnes 85/86 et 86/87.

Le taux de recapture brut est en moyenne de $25,9 \%$, variant assez largement selon les années : respectivement 32,5 et $19,3 \%$ pour les deux campagnes prises en compte.

Légèrement plus élevé pour les femelles que pour les mâles (26.9 contre $23.3 \%$ ), il apparaît largement dépendant de l'état du poisson au moment de son marquage : de $31,5 \%$ en moyenne pour les poissons classés en bon à très bon état au marquage, le taux de recapture tombe en effetà $5.5 \%$ pour les poissons présentant un état moyen (portant des plaies, ulcérations et mycoses)

La recapture des poissons marqués se fait à $80 \%$ dans l'année qui suit l'hiver de marquage. Les recaptures multiples représentent $13 \%$ du total des recaptures; elles sont essentiellement constituées de femelles : ainsi le rapport des sexes (nombre de femelles / nombre de mâles) est-il de 1,8 au moment du marquage, de 2,1 à la première recapture et de 10 pour les recaptures multiples.

Les taux de retour, au piège et à la zone de départ, sont respectivement de

Taux de retour à la zone de départ $1^{\text {re }}$ campagne

$2^{e}$ campagne

Taux de retour au piège

$\begin{array}{cc}50.4 \% & 31,3 \% \\ 30 \% & 20 \%\end{array}$

\section{DISCUSSION - CONCLUSION}

Les résultats obtenus sur le bassin de la Bresle en matière de taux de recapture et de retour de la truite de mer permettent de dégager les grands traits suivants :

\section{- sur le plan méthodologique}

+ les valeurs observées sont assez largement dépendantes du mode de marquage utilisé. Globalement et ainsi que l'ont précédemment souligné plusieurs auteurs $(27,10,17,5)$, le marquage Carlin apparaît moins performant que le cryomarquage puisqu'en moyenne, les taux de recapture et de retour obtenus par le premier sont inférieurs d'un facteur 1,3 à 1,8 à ceux obtenus par le second.

Ces moins bons résultats peuvent être liés à la fois

- à un taux plus élevé de perte de marque. Rappelons que le cryomarquage est toujours associè à une ablation de la nageoire adipeuse qui a permis d'estimer le taux d'effacement du tatouage et de corriger en conséquence les taux de retour; en revanche, aucune correction n'a été faite pour le marquage Carlin, utilisé seul. La tenue de la marque, en partie dépendante de l'expérience de l'équipe de marquage (25), a pu semble-t-il être notablement améliorée grâce à des perfectionnements techniques simples (serrage du nœud à la pince). Les résultats obtenus sur la Bresle semblent d'ailleurs corroborer l'importance de l'expérience de l'équipe de marquage; en effet, la progression constante des taux de recapture et de retour observée pour le marquage par étiquette Carlin (qui aboutit, pour la cohorte 1986, à des résultats équivalents à ceux du cryomarquage) ne peut ètre imputée ni à l'amélioration de l'efficacité du dispositif de piègeage ni à l'accroissement du volume des marques déclarèes par les pêcheurs.

- à une augmentation de la mortalité plus forte chez les smolts étiquetés ainsi que semblent le suggérer les taux de recapture beaucoup plus élevés enregistrés chez les grands smolts. Cette relation entre taille du smolt et taux de recapture, observée à diverses reprises chez le saumon $(4,22,3,8,9)$, serait toutefois liée selon certains auteurs (3) à un problème de perte de marque différentielle.

Ceci étant, le choix du mode de marquage est aussi fonction des objectifs poursuivis: si le cryomarquage apparaît tout à fait adéquat pour l'évaluation des taux de retour à la rivière, avec une lisibilité qui reste bonne plusieurs années après marquage, les étiquettes Carlin sont en revanche nettement plus adaptées pour l'étude des déplacements en mer, les seuls retours lointains provenant en effet de poissons étiquétés (marques mieux détectées par les pêcheurs et facilement déclarables).

+ la qualité des résultats des campagnes de marquage dépend aussi, dans une large mesure. de la capacité de récupération des marques. L'intensification de l'effort de récupération tant par piègeage (à partir de 1984) qu'auprès des pêcheurs (à partir de 1986) s'est d'ailleurs traduite par une progression des taux de recapture et de retour, l'effet de l'information des pêcheurs étant d'ailleurs plus perceptible sur le retour des cryomarques que sur celui des étiquettes. Ceci étant, il semble bien que l'on ait aujourd'hui pratiquement atteint, sur la zone Bresle, le maximum possible en la matière, tout gain supplémentaire d'information ne paraissant désormais pouvoir s'obtenir qu'au prix d'un accroissement démesuré de l'effort d'enquête. 


\section{- sur le plan des résultats}

Ces expériences de marquage ont mis en évidence :

- pour les poissons marqués au stade smolt, des taux de recapture et de retour élevés, relativement homogènes sur les cinq campagnes réalisées; comparés à ceux obtenus sur le saumon atlantique dans les mêmes conditions ( ${ }^{*}$ ), les taux de recapture et de retour de la truite de mer sont régulièrement supérieurs, d'un facteur 1,8 à 2,2 .

- pour les adultes bécards, une importante survie marine post-dévalaison, pouvant varier assez largement d'une année sur l'autre, en relation étroite semble-t-il avec l'état des poissons après le frai.

Toutefois, malgré les corrections apportées, les chiffres obtenus ne constituent que des valeurs minimales en raison essentiellement de la non-déclaration des marques par les pêcheurs et de la difficulté, voire de l'impossibilité, d'estimer correctement les prises réalisées par certaines catégories, tout particulièrement la petite pêche en bateau, amateur et professionnelle.

Le problème se pose en outre de savoir de combien ces taux, calculés sur des poissons marqués, sont inférieurs à ceux de populations naturelles, non marquées et non manipulées. Il est unanimement admis que les manipulations affectent la survie du poisson d'un facteur qui dépend largement des conditions expérimentales et notamment du type de marques utilisées, de l'espèce et des caractéristiques des poissons marqués $(27,10,19,17,12,21,2)$; chez le saumon, où elle a été plus étudiée, cette augmentation de la mortalité se situerait entre 1,8 et $3(24,7,5,21,6)$.

On peut s'attendre à ce que cette mortalité induite soit moins importante chez la truite de mer dont les smolts, plus grands, sont a priori moins vulnérables que ceux du saumon mais elle n'a pu être évaluée pour le moment.

Quoi qu'il en soit, ces bons taux de retour de la truite de mer sont à relier vraisemblablement:

- d'une part à la taille élevée des smolts

- d'autre part à une migration marine de relativement faible amplitude, comparée à celle du saumon. Les valeurs obtenues se rapprochent d'ailleurs beaucoup de celles observées chez les saumons à migration marine modérée : castillons islandais ou saumons de la Baltique $(7,13)$. délicate :

Ceci dit, la comparaison avec les résultats rencontrés en bibliographie est généralement

- soit parce qu'il s'agit de poissons différents : la majeure partie des données disponibles concerne le saumon

- soit parce que les conditions expérimentales, au marquage et à la recapture, ou les objectifs poursuivis diffèrent sensiblement $(15,16,14,1,11,20,18,23,26)$.

L'analyse est de plus compliquée par la flexibilité comportementale de la truite de mer: retours en tant que finnocks, frais multiples,...

Les résultats obtenus sur le bassin de la Bresle apportent une utile contribution à la connaissance des taux de retour de la truite de mer et par suite, de sa dynamique de population, tant au niveau national où il n'existe pas de chiffres sur le sujet, qu'au niveau international où les données concernant la truite de mer restent rares.

\section{BIBLIOGRAPHIE}

(01) ANONYME, 1981. Annual Report n XXV (Year 1980). Salm. Res. Trust Ireland Inc., $43 \mathrm{p}$.

(02) CHADWICK E.M.P., REDDIN D.G. and BURFITT R.F., 1985. Fishing and natural mortality rates for $1 \mathrm{SW}$ Atlantic salmon (Salmo salar L.). I.C.E.S. CM/M, 18, 11 p.

(03) EISNER R.A., RITTER J.A., 1979. The canadian Atlantic salmon (Salmo salar) smolt tag and its recovery system : a review. I.C.E.S. CM/M, 24, 12 p.

(04) GUDJONSON T., 1970. The releases and return of tagged salmon at Kollafjordur, Iceland. I.C.E.S. CM/M, 6,6 p.

(") FOURNEL F., EUZENAT G., données non publiées.

Taux de recapture et de retour obtenus à partir de smolts sauvages cryomarqués - trois campagnes réalisées :

- taux de recapture : $4,5 \%$

- taux de retour au piège : $5,5 \%$

- taux de retour à la zone de départ : $6,8 \%$. 
(05) HANSEN L.P., 1981. Returns of Carlin tagged, fin-clipped and unmarked wild smolts of Atlantic salmon (Salmo salar L.) from the river Imsa, SW Norway. I.C.E.S. CM/M, 6, 6 p.

(06) HAY D.W., 1985. Overwinter post-tagging mortality and tag loss among autumn emigrating juvenile Atlantic salmon (Salmo salar L.) held overwinter in tanks. I.C.E.S. CM/M, 13, 7 p.

(07) ISAKSSON A., 1980. Salmon Ranching in Iceland : 131 - 156. In THORPE J.E. (Ed.) SALMON RANCHING, Academic Press London, $440 \mathrm{p}$.

(08) ISAKSSON A., 1982. Returns of microtagged Atlantic salmon (Salmo salar) to the Kollafjordur experimental fish farm in $1976-79$ tagging experiments. I.C.E.S. CM/M, 34, 9 p.

(09) ISAKSSON A., 1982. Returns of microtagged Atlantic salmon (Salmo salar) of Kollafjordur stock to three different salmon ranching facilities. I.C.E.S. CM/M, 35, $9 \mathrm{p}$.

(10) ISAKSSON A., BERGMAN P.K., 1979. An evaluation of two tagging methods and survival rates of different ages and treatment groups of hatchery reared Atlantic salmon smolts. J. Agr. Res. Icel., 10, $1: 74-99$.

(11) JENSEN K.W., 1968. Sea trout (Salmo trutta) of the river Istra. Western Norway. Inst. Freshwat. Res. Drottningholm, $48: 187-213$.

(12) JENSEN K.W., 1981. Survival estimates of wild smolts of Atlantic salmon from river Imsa, S.W. Norway. I.C.E.S. CM/M, 14, 5 p.

(13) LARSSON P.O., 1980. Smolt rearing and the Baltic salmon Fishery: 157 - 186. In THORPE J.E. (Ed.) SALMON RANCHING. Academic Press London, $440 p$

(14) LARSSON P.O., STEFFNER N.G., LARSSON H.O., ERIKSSON C., 1979. Review of swedish sea trout (Salmo trutta L.) stocks based on results of tagging experiments. Salmon Research Institute Report, 1979 (2), $31 \mathrm{p}$

(15) PEMBERTON R., 1976. Sea trout in North Argyll sea lochs, population, distribution and movements. J. Fish Biol., 9 : 157 - 179.

(16) PIGGINGS D.J., 1976. Stock production, survival rates and life history of the sea trout of the Burrishoole river system. Salm. Res. Trust Ireland Inc., Ann. Rep. $20: 45$ - 57.

(17) PIGGINS D.J., 1980. Salmon Ranching in Ireland, 187 - 198. In THORPE J.E. (Ed.) SALMON RANCHING, Academic Press London, 440 p.

(18) PIGGINS D.J., 1984. Sea trout in the Burrishoole system. Communication Symposium "The Biology of the sea trout" - Plas Menai, Octobre 84. Ed. by LE CREN E.D.

(19) PORTER T.R., 1979. A review of factors affecting the rate of return of tagged Atlantic salmon and effects of tagging. I.C.E.S. CM/M, 15, $17 \mathrm{p}$.

(20) PRATTEN D.J., SHEARER W.M., 1983. The migrations of North Esk sea-trout. Fish. Mgmt., 14(3): $99-113$.

(21) PROUZET P., 1983. Le pacage en mer du Saumon atlantique en Europe. La Pêche Maritime, 202, $202-208$.

(22) RITTER J.A., 1973. Preliminary observation on the influence of smolt size on tag return rate and age at first maturity of Atlantic salmon (Salmo salar). I.C.N.A.F. Res. Doc. 73/67, 10 p.

(23) SAMBROOK H., 1984. Homing specificity among sea-trout of the river Fowery system. - Validation of scale reading using recaptured sea trout. Communication Symposium "The Biology of the Sea trout" - Plas Menai, Octobre 1984. Ed. by LE CREN E.D.

(24) SAUNDERS R.L., ALLEN K. R., 1967. Effects of tagging and fin-clipping on the survival and growth of Atlantic salmon between smolt and adult stages. J. Fish. Res. Bd. Canada, 24(12) $2595-2611$.

(25) SHEARER W.M., 1977. Factors affecting the estimation of the survival of tagged Atlantic salmon smolts as returning adults. I.C.E.S. CM/M, 13, 4 p.

(26) SHEARER W.M., 1984. Sea trout studies in the North Esk. Communication Symposium "The Biology of the Sea trout" - Plas Menai, Octobre 1984. Ed. by LE CREN E.D.

(27) VICKEAS K.U., 1977. Survival of smolts from the river Bush. I.C.E.S. CM/M., 15, 4 p. 


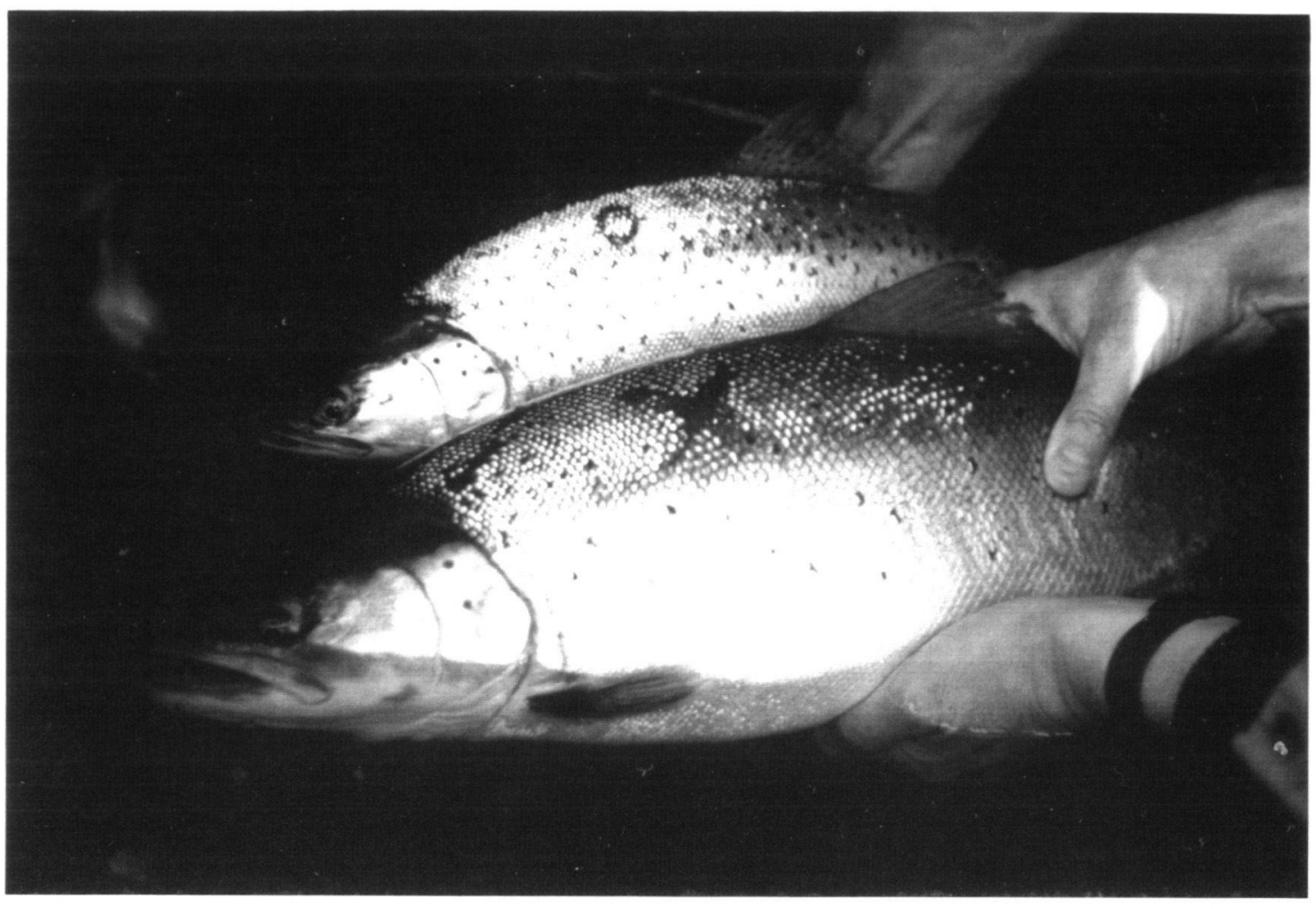

Photo 1 : Truites de mer cryomarquées, capturées le 25/05/87 dans le dispositif de piègeage de Eu (rivière BRESLE)

Marque $O$ (printemps 86) : âge de mer I+ $-526 \mathrm{~mm} / 1,910 \mathrm{~kg}$

Marque X (printemps 85) : âge de mer II+ $-690 \mathrm{~mm} / 5,410 \mathrm{~kg}$

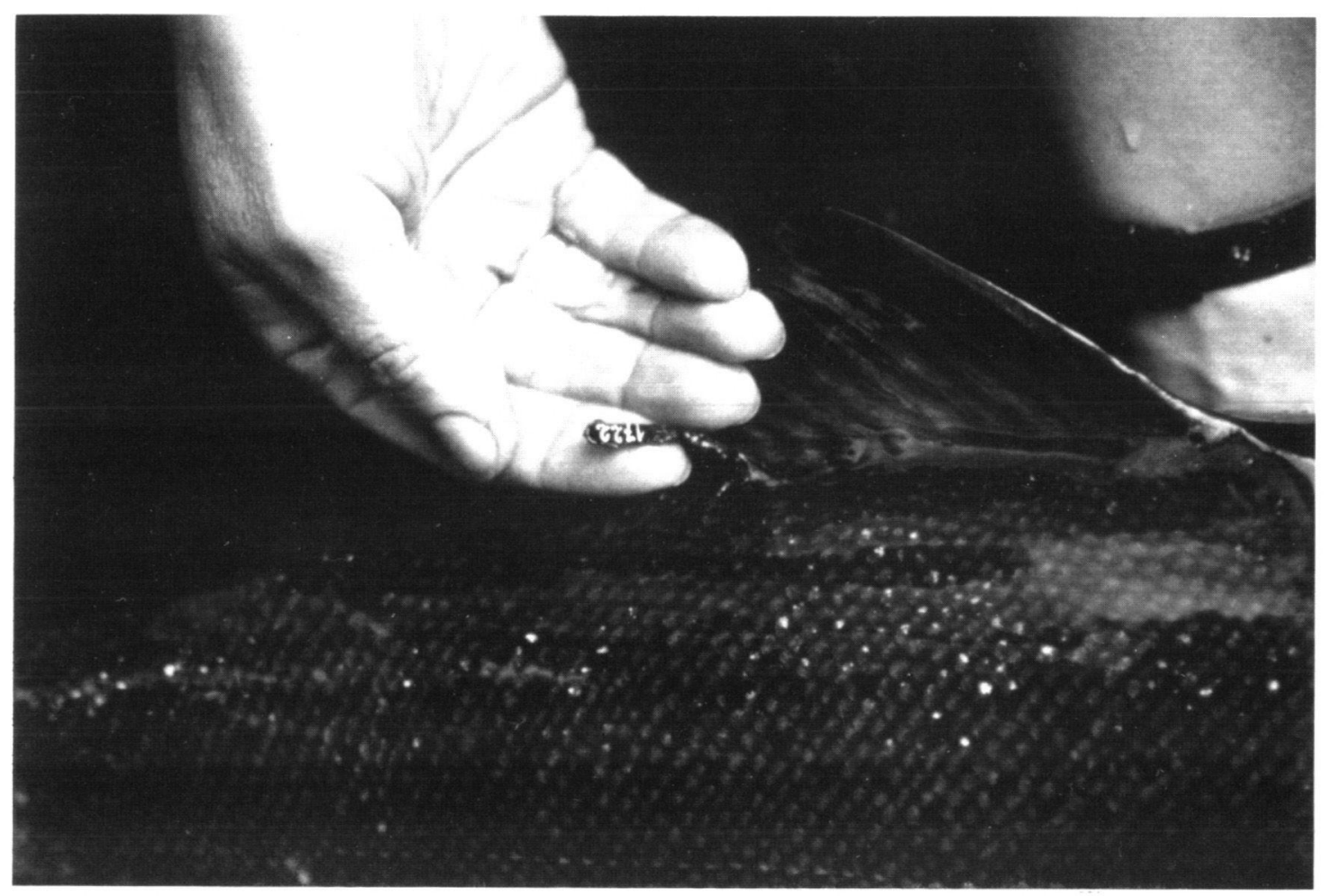

Photo 2 : Truite de mer porteuse d'une étiquette Carlin, capturée le 23/06/89 dans le dispositif de piègeage de Eu (rivière BRESLE) : $720 \mathrm{~mm} / 4,210 \mathrm{~kg}$ - marquée le 11/01/88 : $560 \mathrm{~mm} / 1,500 \mathrm{~kg}$. 